\title{
Normal mode analysis of the fully developed free convection flow in a vertical slot with open to capped ends
}

Journal Article

Author(s):

Magyari, E.

Publication date:

2007-06

Permanent link:

https://doi.org/10.3929/ethz-b-000067630

Rights / license:

In Copyright - Non-Commercial Use Permitted

Originally published in:

Heat and Mass Transfer 43(8), https://doi.org/10.1007/s00231-006-0162-2 


\title{
Normal mode analysis of the fully developed free convection flow in a vertical slot with open to capped ends
}

\author{
E. Magyari
}

Received: 2 March 2006/ Accepted: 16 June 2006/ Published online: 22 July 2006

(C) Springer-Verlag 2006

\begin{abstract}
The fully developed free convection flow in a differentially heated vertical slot with open to capped ends investigated recently by Bühler (Heat Mass Transf 39:631-638, 2003) and Weidman (Heat Mass Transf Online First, February 2006) is revisited in this paper. A new method of solution of the corresponding fourth order boundary value problem, based on its reduction to "normal modes" by a complex matrix similarity transformation is presented. As a byproduct of the method, some invariant relationships involving the heat flux and the shear stress in the flow could be found.
\end{abstract}

\section{Introduction}

The present report is closely related to the recent papers by Bühler [1] and Weidman [2] in which the conduction [1] and convection [2] regime of the free convection flow in a differentially heated tall vertical slot with open to capped ends has been investigated. For the quasi-static transition from the open to the capped end situation, in both cases a continuum of solutions have been found.

The fully developed steady convection flow is governed by the fourth order system of coupled differential equations

E. Magyari $(\bowtie)$

Institute of Building Technology,

ETH Zürich, Wolfgang-Pauli-Str.1,

CH-8093 Zurich, Switzerland

e-mail: magyari@hbt.arch.ethz.ch $u^{\prime \prime}+G \theta=0, \quad \theta^{\prime \prime}-E u=0$

along with the boundary conditions

$u( \pm 1 / 2)=0, \theta(-1 / 2)=-\theta_{0}, \theta(1 / 2)=1-\theta_{0}$,

where $u=u(y)$ and $\theta=\theta(y)$ are the dimensionless velocity and temperature fields, $G$ and $E$ the Grashof and Elder numbers, respectively, and the primes denote differentiation with respect to the dimensionless horizontal coordinate $-0.5 \leq y \leq+0.5$ of the slot [2]. We mention that the boundary conditions given here in (2) are the intended boundary conditions for Eqs. (12) of P.D. Weidman (private communication).

The features of the fully developed flow are independent of the vertical coordinate $x$. The variation range of the dimensionless temperature parameter $\theta_{0}$ is $0 \leq \theta_{0} \leq 1 / 2$, such that the value $\theta_{0}=0$ corresponds to the open and $\theta_{0}=1 / 2$ to the capped slot, respectively. The intermediate values of $\theta_{0}$ are associated with permeable caps of which porosity decreases from 1 to 0 as $\theta_{0}$ increases from 0 to $1 / 2$. The coordinate system is chosen symmetrically, [2], such that $y=-1 / 2$ corresponds to the cold and $y=+1 / 2$ hot wall of the slot, in agreement with the boundary conditions (2).

The continuum of convection solutions has been obtained by Weidman, [2], by eliminating the velocity $u$ from Eqs. (1) and solving the fourth order boundary value problem resulting in this way for the temperature variable $\theta$. The aim of the present paper is to report a new method of solution which is based on the reduction of the problem (1), (2) to its "normal modes" (known from lattice dynamics) by applying a complex matrix similarity transformation. This quite general matrix diagonalization method allows one to find the 
solutions without increasing the order of equations (from 2 to 4 ). In addition, it makes possible a transparent and powerful complex analysis of the mathematical and physical features of the problem. As a byproduct of the method, some new invariant relationships involving the heat flux and the shear stress in the flow will be reported.

\section{Solution by reduction to normal modes}

We first transcribe Eqs. (1) to the matrix-differential form

$\left(\begin{array}{cc}L & G \\ -E & L\end{array}\right)\left(\begin{array}{c}u \\ \theta\end{array}\right)=0$

where $L=\mathrm{d}^{2} / \mathrm{d} y^{2}$. Then we subject Eq. (3) to the matrix-similarity transformation

$S^{-1}\left(\begin{array}{cc}L & G \\ -E & L\end{array}\right) S S^{-1}\left(\begin{array}{c}u \\ \theta\end{array}\right)=0$,

where $S$ is some non-singular $2 \times 2$ matrix with the constant matrix elements $S_{i j}$ and $S^{-1}$ stands for the inverse matrix of $S$. Now further denote

$S^{-1}\left(\begin{array}{c}u \\ \theta\end{array}\right)=\left(\begin{array}{l}Z_{1} \\ Z_{2}\end{array}\right)$ which in turn yields $\left(\begin{array}{c}u \\ \theta\end{array}\right)=S\left(\begin{array}{l}Z_{1} \\ Z_{2}\end{array}\right)$

and chose $S$ such that in Eq. (4) the transformed matrix $S^{-1}(\ldots) S$ becomes diagonal. This requirement is satisfied by the choice

$S=\left(\begin{array}{cc}i \sqrt{G / E} & 1 \\ 1 & i \sqrt{E / G}\end{array}\right), S^{-1}=-\frac{1}{2}\left(\begin{array}{cc}i \sqrt{E / G} & -1 \\ -1 & i \sqrt{G / E}\end{array}\right)$.

The diagonalized Eq. (4) reads

$$
\left(\begin{array}{cc}
L+\omega_{1}^{2} & 0 \\
0 & L+\omega_{2}^{2}
\end{array}\right)\left(\begin{array}{l}
Z_{1} \\
Z_{2}
\end{array}\right)=0
$$

where

$\omega_{1}=(i-1) m, \omega_{2}=(i+1) m, m=(G E / 4)^{1 / 4}, i=\sqrt{-1}$.

The matrix equation (7) yields for $Z_{1}$ and $Z_{2}$ the decoupled differential equations

$Z^{\prime \prime}{ }_{1,2}=-\omega_{1,2}^{2} Z_{1,2}$
Equations (9) may formally be seen as the differential equations of motion of two independent linear oscillators of "mass" $=1$ with (complex) frequencies $\omega_{1}$ and $\omega_{2}$, and with $y$ being the time variable of motion. Accordingly, the general solutions for the two "normal oscillation modes" $Z_{1}$ and $Z_{2}$ can be written down immediately,

$Z_{1,2}(y)=A_{1,2} \sin \left(\omega_{1,2} y+\alpha_{1,2}\right)$,

where $A_{1,2}$ and $\alpha_{1,2}$ are integration constants. Thus the solution $(u, \theta)$ of our boundary value problem is obtained from the second matrix equation (5) in terms of the "normal modes" $Z_{1}$ and $Z_{2}$ in the form

$\begin{aligned} u & =i(G / E)^{1 / 2} Z_{1}+Z_{2}, \\ \theta & =i(E / G)^{1 / 2} Z_{2}+Z_{1} .\end{aligned}$

Boundary conditions (2) lead via Eqs. (10) and (11) to the following conditions for $Z_{1}$ and $Z_{2}$ :

$$
\begin{aligned}
Z_{1}(-1 / 2)= & -\theta_{0} / 2, Z_{1}(1 / 2)=\left(1-\theta_{0}\right) / 2, \\
Z_{2}(-1 / 2)= & i(G / E)^{1 / 2} \theta_{0} / 2, Z_{2}(1 / 2)= \\
& -i(G / E)^{1 / 2}\left(1-\theta_{0}\right) / 2 .
\end{aligned}
$$

Thus we easily obtain for the integration constants the expressions

$\alpha_{1,2}=\arctan \left[\left(1-2 \theta_{0}\right) \tan \left(\omega_{1,2} / 2\right)\right]$

$A_{1}=\frac{1}{4 \cos \alpha_{1} \sin \left(\omega_{1} / 2\right)}, A_{2}=-\frac{i(G / E)^{1 / 2}}{4 \cos \alpha_{2} \sin \left(\omega_{2} / 2\right)}$.

In this way we obtain for the fields $(u, \theta)$ the explicit results

$$
\begin{aligned}
& u(y)=\frac{i}{4} \sqrt{\frac{G}{E}}\left[\frac{\sin \left(\omega_{1} y+\alpha_{1}\right)}{\cos \alpha_{1} \sin \left(\omega_{1} / 2\right)}-\frac{\sin \left(\omega_{2} y+\alpha_{2}\right)}{\cos \alpha_{2} \sin \left(\omega_{2} / 2\right)}\right], \\
& \theta(y)=\frac{1}{4}\left[\frac{\sin \left(\omega_{1} y+\alpha_{1}\right)}{\cos \alpha_{1} \sin \left(\omega_{1} / 2\right)}+\frac{\sin \left(\omega_{2} y+\alpha_{2}\right)}{\cos \alpha_{2} \sin \left(\omega_{2} / 2\right)}\right] .
\end{aligned}
$$

For convenience, in Table 1 of Appendix A the values of $\alpha_{1,2}, A_{1,2}$ and $Z_{1,2}$ have been summarized for the limiting cases of the open $\left(\theta_{0}=0\right)$ and capped $\left(\theta_{0}=1 / 2\right)$ slot, respectively.

\section{Complex analysis}

It is easy to see that under the operation of complex conjugation, which we denote by the symbol *, the quantities introduced in Sect. 2 behave as follows: 
$\omega_{2}^{*}=-\omega_{1}, \alpha_{2}^{*}=-\alpha_{1}, A_{2}^{*}=-i(G / E)^{1 / 2} A_{1}$,

$Z_{2}^{*}=i(G / E)^{1 / 2} Z_{1}$.

In this way the expressions (11) of the velocity and temperature fields become

$$
\begin{aligned}
u(y) & =\frac{i G}{2 m^{2}}\left(Z_{1}-Z_{1}^{*}\right), \\
\theta(y) & =Z_{1}+Z_{1}^{*},
\end{aligned}
$$

or

$$
\begin{aligned}
& u(y)=-\frac{G}{m^{2}} \operatorname{Im}\left[Z_{1}(y)\right] \\
& \theta(y)=2 \operatorname{Re}\left[Z_{1}(y)\right]
\end{aligned}
$$

where Im and $\mathrm{Re}$ stand for the imaginary and real part of the complex normal mode $Z_{1}$. Equations (18) show explicitly that $u$ and $\theta$ are real quantities (as expected). At the same time Eqs. (18) show that $u$ and $\theta$ can be expressed in terms of the (complex) normal mode $Z_{1}$ alone. This is a direct consequence of the features (16) and represents a substantial advantage of the complex calculus. Hence we may ignore the index 1 in favour of the simpler notation $\omega_{1} \equiv \omega=(i-1) m$.

The compact expressions (18) for the fields $u$ and $\theta$ and are equally suitable for both symbolical and numerical calculations (by using standard library programs). As a first example, for the dimensionless planar volume flux of the flow,

$Q=\int_{-1 / 2}^{1 / 2} u \mathrm{~d} y$

we easily obtain the expression

$Q=\frac{G}{m^{2}}\left(\theta_{0}-\frac{1}{2}\right) \operatorname{Im}\left[\frac{1}{\omega} \tan \frac{\omega}{2}\right]$.

This agrees with the expression given in Eq. (20) of Weidman [2] when the denominator term $\chi^{2}+1$ is replaced by $\Omega^{2}+1$.

As it is well known, every function $f=f(x)$ can be represented as a sum of a symmetric and antisymmetric function (more precisely of an even and odd function of $x), f(x)=f_{s}(x)+f_{a}(x)$ where $2 f_{s}(x)=f(x)+f(-x)$ and $2 f_{a}(x)=f(x)-f(-x)$. In the present case, with the aid of Eqs. (10)-(18) we obtain

$$
\begin{aligned}
& u(y)=-\frac{G}{2 m^{2}} \operatorname{Im}\left[\left(\frac{1}{2}-\theta_{0}\right) \frac{\cos \omega y}{\cos (\omega / 2)}+\frac{1}{2} \frac{\sin \omega y}{\sin (\omega / 2)}\right] \\
& \theta(y)=\operatorname{Re}\left[\left(\frac{1}{2}-\theta_{0}\right) \frac{\cos \omega y}{\cos (\omega / 2)}+\frac{1}{2} \frac{\sin \omega y}{\sin (\omega / 2)}\right]
\end{aligned}
$$

Now all the quantities of physical and engineering interest, namely the volume flux $Q$, the dimensionless skin frictions $s_{ \pm}=u^{\prime}( \pm 1 / 2)$ and the dimensionless wall heat fluxes $q_{ \pm}=-\theta^{\prime}( \pm 1 / 2)$, can be calculated from Eqs. (20) and (21) in terms of the parameters of the problem easily. The results are

$$
\begin{aligned}
Q & =\frac{G}{2 m^{3}}\left(\theta_{0}-\frac{1}{2}\right) \frac{\sin m-\sinh m}{\cos m+\cosh m}, \\
s_{ \pm} & \equiv u^{\prime}( \pm 1 / 2) \\
& =-\frac{G}{4 m}\left[\frac{\sinh m-\sin m}{\cosh m-\cos m} \pm\left(1-2 \theta_{0}\right) \frac{\sinh m+\sin m}{\cosh m+\cos m}\right]
\end{aligned}
$$

$$
\begin{aligned}
q_{ \pm} & \equiv-\theta^{\prime}( \pm 1 / 2) \\
& =-\frac{m}{2}\left[\frac{\sinh m+\sin m}{\cosh m-\cos m} \pm\left(1-2 \theta_{0}\right) \frac{\sinh m-\sin m}{\cosh m+\cos m}\right] .
\end{aligned}
$$

The content of Eqs. (21) and (22) is the same as those of Eqs. (13)-(20) of Weidman [2]. For convenience, in Table 2 of Appendix B the quantities $u(y), \theta(y), Q, s_{ \pm}$ and $q_{ \pm}$have been summarized for the limiting cases of open $\left(\theta_{0}=0\right)$ and capped $\left(\theta_{0}=1 / 2\right)$ slots, respectively.

The limiting case of steady convection in the conduction regime which corresponds to $E \rightarrow 0$, can also easily be obtained from the above equations. Indeed, according to Eqs. (8), $E \rightarrow 0$ implies $m \rightarrow 0$ which in turn implies $\omega \rightarrow 0$. Thus, expanding Eqs. (21)-(24) in Taylor series of powers of $m$, we obtain in the conduction regime limit $m=0$ equations (38-44) listed in Appendix C.

\section{Invariant quantities}

In addition to its quite general applicability and transparency, the "normal-mode"-approach presented above facilitates the determination of some invariant (coordinate independent) relationships between the shear stress and the heat flux in the fluid. Indeed, the total mechanical energies $Z_{n}{ }^{2} / 2+\omega_{n}{ }^{2} Z_{n}{ }^{2} / 2, n=1,2$ of the individual "normal oscillators" are conserved quantities (i.e. independent of "time" y). Having in 
mind Eqs. (10), we see that these constants are equal to $\omega_{n}{ }^{2} A_{n}{ }^{2} / 2$ such that the two "integrals of motion" are

$Z_{n}^{\prime 2}+\omega_{n}^{2} Z_{n}^{2}=\omega_{n}^{2} A_{n}^{2}, n=1,2$,

where, according to the second equations (5) and (6),

$Z_{1}=\frac{1}{2}\left(\theta-i \sqrt{\frac{E}{G}} u\right), Z_{2}=\frac{1}{2}\left(u-i \sqrt{\frac{G}{E}} \theta\right)$.

Now, taking into account the fact that the two normal modes $Z_{1,2}$ are related to each other by the operation of complex conjugation (see Eqs. (16)), it is sufficient to consider only one of Eqs. (25). Thus, choosing the normal mode " 1 " and dropping its subscript for simplicity of notation, we obtain from Eqs. (25) and (26)

$$
\left(\theta^{\prime}-i \sqrt{\frac{E}{G}} u^{\prime}\right)^{2}+\omega^{2}\left(\theta-i \sqrt{\frac{E}{G}} u\right)^{2}=4 \omega^{2} A^{2} .
$$

These relationships between the dimensionless shear stress $u^{\prime}(y)$ and the dimensionless heat flux $\theta^{\prime}(y)$ hold for all values $-0.5 \leq y \leq 0.5$ of the coordinate $y$, as anticipated above. Of practical interest are obviously the values of these quantities at the walls. Thus, substituting in Eq. (27) $y=1 / 2$ and $y=-1 / 2$, and taking into account the boundary conditions (2), we obtain that between the corresponding skin frictions $u^{\prime}( \pm 1 / 2) \equiv s_{ \pm}$and wall heat fluxes $\theta^{\prime}( \pm 1 / 2) \equiv q_{ \pm}$the following relationships hold

$$
\begin{aligned}
& \left(q_{+}+i \sqrt{\frac{E}{G}} s_{+}\right)^{2}+\omega^{2}\left(1-\theta_{0}\right)^{2}=4 \omega^{2} A^{2}, \\
& \left(q_{-}+i \sqrt{\frac{E}{G}} s_{-}\right)^{2}+\omega^{2} \theta_{0}^{2}=4 \omega^{2} A^{2} .
\end{aligned}
$$

Furthermore, we obtain by subtraction

$$
\left(q_{+}+i \sqrt{\frac{E}{G}} s_{+}\right)^{2}-\left(q_{-}+i \sqrt{\frac{E}{G}} s_{-}\right)^{2}=\omega^{2}\left(2 \theta_{0}-1\right) .
$$

Having in mind that $\omega^{2}=-2 \mathrm{im}^{2}$ and identifying the real and imaginary parts of Eq. (29) we deduce the relationships

$$
\frac{q_{+}^{2}-q_{-}^{2}}{E}=\frac{s_{+}^{2}-s_{-}^{2}}{G} \text { or } \frac{q_{+}^{2}}{E}-\frac{s_{+}^{2}}{G}=\frac{q_{-}^{2}}{E}-\frac{s_{-}^{2}}{G},
$$

$q_{+} s_{+}-q_{-} s_{-}=\left(\frac{1}{2}-\theta_{0}\right) G$

Equations (30) and (31) represent interesting connections between the heat fluxes and shear stresses at the two walls of the slot and show their connection to the physical parameters $E, G$ and $\theta_{0}$ of the problem. They are somewhat reminiscent of the classical Reynolds analogy resulting from the momentum and temperature boundary layer equations at unity Prandtl number.

\section{Integral momentum and energy balance}

In the preceding section the invariant relationship (27) between the dimensionless shear stress $u^{\prime}(y)$ and the dimensionless heat flux $\theta^{\prime}(y)$ has been established which, when specified for the walls, yields several relationships between the skin frictions $s_{ \pm}=u^{\prime}( \pm 1 / 2)$ and wall heat fluxes $q_{ \pm}=\theta^{\prime}( \pm 1 / 2)$ [see Eqs. (28)-(31)]. Further interesting relationships between the quantities $s_{ \pm}, q_{ \pm}$and $Q$ can be obtained by integrating Eqs. (1) between the walls. By doing so we easily obtain

$s_{-}-s_{+}=G \theta_{m}$,

$q_{-}-q_{+}=E Q$,

where $Q$ is the planar volume flux (19) and $\theta_{m}$ the average temperature

$\theta_{m}=\int_{-1 / 2}^{1 / 2} \theta \mathrm{d} y$

Thus, Eqs. (32), (33) and (30) yield the relationship

$\frac{\theta_{m}}{Q}=\frac{q_{+}+q_{-}}{s_{+}+s_{-}}$

while from Eqs.(32) and (23) for the average temperature the explicit expression

$\theta_{m}=\frac{G}{m}\left(\frac{1}{2}-\theta_{0}\right) \frac{\sinh m+\sin m}{\cosh m+\cos m}$

results. According to Eqs. (32) and (33) the change of the skin friction across the slot equals the average temperature $\theta_{m}$ scaled with the Grashof number and the change of the wall heat flux equals the planar volume flux $Q$ scaled with the Elder number, respectively. 
The main physical message emerges from the integral energy balance equation (33). Indeed, the slot can be viewed as a heat transfer device which transfers heat from the warm right wall to the cold left one. In this sense $q_{-}-q_{+}$, which on account of Eqs. (24) is given by

$q_{-}-q_{+}=2 m\left(\frac{1}{2}-\theta_{0}\right) \frac{\sinh m-\sin m}{\cosh m+\cos m}$

represents the heat lost during the transfer process. Obviously, this amount of heat is "washed away" by the convecting fluid, so by this physical mechanism, the transmission loss $q_{-}-q_{+}$is proportional to the volume flux $Q$. Accordingly, for the capped slot $\left(\theta_{0}=1 / 2\right)$ where $Q=0$, we obtain $q_{-}=q_{+}$(in agreement with Table 2 of Appendix B). In other words, the closed slot is a loss-free heat transfer device. The same holds for the conduction limit $m=0$, regardless the value of $\theta_{0}$ (see Appendix C). This circumstance can also be seen in Fig. 1 where the transmission heat loss $q_{-}-q_{+}$is plotted as function of the parameter $m$. The larger $m$, the larger the heat lost by transmission through the slot for any specified value $\theta_{0} \neq 1 / 2$. For a specified $m$, however, $q_{-}-q_{+}$decreases as $\theta_{0}$ approaches the value $\theta_{0}=1 / 2$ corresponding to capped end situation.

\section{Summary and conclusions}

The convection model of the fully developed flow in a differentially heated vertical slot with open to capped ends proposed recently by Weidman [2] has been examined in this paper. The main results can be summarized as follows.

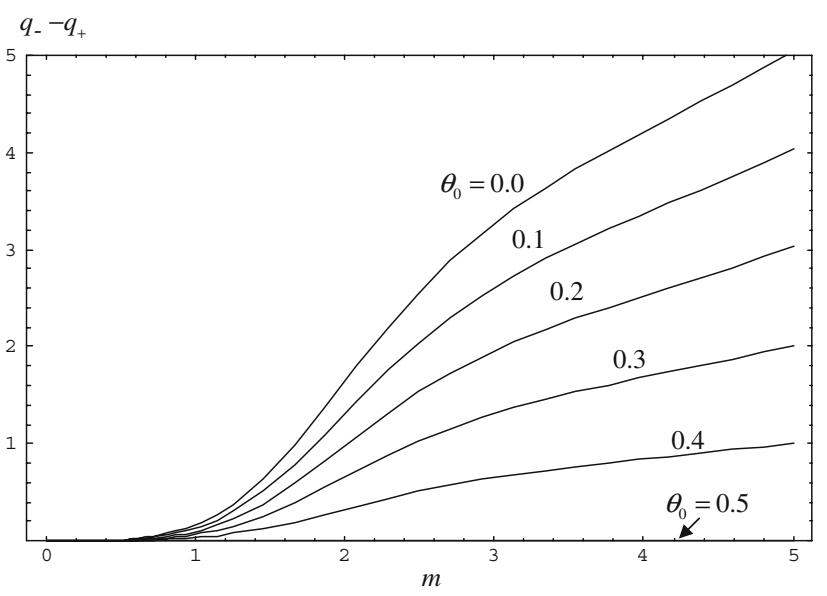

Fig. 1 Heat transmission loss $q_{-}-q_{+}$of the slot plotted as function of $m$ for six different values of $\theta_{0}$
1. A new method of solution of the corresponding fourth order boundary value problem, based on its reduction to "normal modes" (known from the lattice dynamics) by a complex matrix similarity transformation, has been presented. The use of complex analysis confers to this method a high degree of transparency as well as an algebraic and computational efficiency (similarly to its use in electrical network theory and in heat conduction of harmonically excited slabs). As a byproduct, some new invariant relationships involving the heat flux and the shear stress in the flow could be found.

2. It has been emphasized that the dimensionless temperature field $\theta(y)$ and the velocity field scaled by the Grashof number, $u(y) / G$, are characterized by only two physical parameters: the temperature parameter $0 \leq \theta_{0} \leq 0.5$ (such that the lower bound of $\theta_{0}$ corresponds to the open and the upper one to the capped slot) and a second parameter $m$ which depends on the product of the Grashof and Elder numbers in the form $m=(G E / 4)^{1 / 4}$, [2].

3. The capped slot $\left(\theta_{0}=0\right)$ is an ideal heat transfer device; it transfers heat from the warm to the cold wall without any loss. The same holds for the conduction limit $m=0$, regardless the value of $\theta_{0}$. The larger $m$, the larger the heat lost by transmission through the slot for any specified value $\theta_{0} \neq 1 / 2$ (see Fig. 1).

Acknowledgments The author is indebted to Prof. P. D. Weidman of University of Colorado at Boulder for prolific discussions during the preparation of this paper.

\section{Appendix A}

Table 1 Overview of the values of $\alpha_{1,2}, A_{1,2}$ and $Z_{1,2}$ for the limiting cases of open $\left(\theta_{0}=0\right)$ and capped $\left(\theta_{0}=1 / 2\right)$ slot, respectively

\begin{tabular}{lll}
\hline & Open slot: $\theta_{0}=0$ & Capped slot: $\theta_{0}=1 / 2$ \\
\hline$\alpha_{1}$ & $\frac{\omega_{1}}{2}$ & 0 \\
$\alpha_{2}$ & $\frac{\omega_{2}}{2}$ & 0 \\
$A_{1}$ & $\frac{1}{2 \sin \omega_{1}}$ & $\frac{1}{4 \sin \left(\omega_{1} / 2\right)}$ \\
$A_{2}$ & $-\frac{i(G / E)^{1 / 2}}{2 \sin \omega_{2}}$ & $-\frac{i(G / E)^{1 / 2}}{4 \sin \left(\omega_{2} / 2\right)}$ \\
$Z_{1}$ & $\frac{\sin \omega_{1}(y+0.5)}{2 \sin \omega_{1}}$ & $\frac{\sin \omega_{1} y}{4 \sin \left(\omega_{1} / 2\right)}$ \\
$Z_{2}$ & $-i\left(\frac{G}{E}\right)^{1 / 2} \frac{\sin \omega_{2}(y+0.5)}{2 \sin \omega_{2}}$ & $-i\left(\frac{G}{E}\right)^{1 / 2} \frac{\sin \omega_{2} y}{4 \sin \left(\omega_{2} / 2\right)}$ \\
\hline
\end{tabular}

The values of $\omega_{1,2}$ are given by Eqs. (8) 


\section{Appendix B}

Table 2 Overview of the expressions of $u(y), \theta(y), Q, s_{ \pm}$and $q_{ \pm}$ for the limiting cases of open $\left(\theta_{0}=0\right)$ and capped $\left(\theta_{0}=1 / 2\right)$ slot, respectively

\begin{tabular}{|c|c|c|}
\hline & Open slot: $\theta_{0}=0$ & Capped slot: $\theta_{0}=1 / 2$ \\
\hline$u(y)$ & $\begin{array}{c}-\frac{G}{2 m^{2}} \operatorname{Im}\left[\frac{\sin \omega(y+0.5)}{\sin \omega}\right] \\
\text { (non-symmetric) }\end{array}$ & $\begin{aligned}- & \frac{G}{4 m^{2}} \operatorname{Im}\left[\frac{\sin \omega y}{\sin (\omega / 2)}\right] \\
& \text { (antisymmetric) }\end{aligned}$ \\
\hline$\theta(y)$ & $\begin{array}{l}\operatorname{Re}\left[\frac{\sin \omega(y+0.5)}{\sin \omega}\right] \\
\text { (non-symmetric) }\end{array}$ & $\begin{array}{l}\frac{1}{2} \operatorname{Re}\left[\frac{\sin \omega y}{\sin (\omega / 2)}\right] \\
\quad \text { (antisymmetric) }\end{array}$ \\
\hline$Q$ & $\frac{G}{4 m^{3}} \frac{\sinh m-\sin m}{\cosh m+\cos m}$ & 0 \\
\hline$s_{+}$ & $\frac{G}{4 m} \frac{\sinh 2 m-\sin 2 m}{\cos ^{2} m-\cosh ^{2} m}$ & $s_{+}=s_{-}=-\frac{G}{4 m} \frac{\sinh m-\sin m}{\cosh m-\cos m}$ \\
\hline$s_{-}$ & $\frac{G}{2 m} \frac{\cos m \sinh m-\sin m \cosh m}{\cos ^{2} m-\cosh ^{2} m}$ & \\
\hline$q_{+}$ & $-\frac{m}{2} \frac{\sinh 2 m+\sin 2 m}{\cosh ^{2} m-\cos ^{2} m}$ & $q_{+}=q_{-}=-\frac{m}{2} \frac{\sinh m+\sin m}{\cosh m-\cos m}$ \\
\hline$q_{-}$ & $-m \frac{\sin m \cosh m+\cos m \sinh m}{\cosh ^{2} m-\cos ^{2} m}$ & \\
\hline
\end{tabular}

\section{Appendix C}

The conduction limit $(m=0)$ is obtained as the leading order terms of the following equations obtained by Taylor expansions of Eqs. (21)-(24) to powers of $m$.

$u(y)=\frac{G}{6}\left(\frac{1}{4}-y^{2}\right)\left[y+3\left(\frac{1}{2}-\theta_{0}\right)\right]$, $\theta(y)=\frac{1}{2}-\theta_{0}+y$,

$Q=\frac{G}{12}\left(\frac{1}{2}-\theta_{0}\right)$,

$s_{+} \equiv u^{\prime}(+1 / 2)=\frac{G}{2}\left(\theta_{0}-\frac{2}{3}\right)$,

$s_{-} \equiv u^{\prime}(-1 / 2)=-\frac{G}{2}\left(\theta_{0}-\frac{1}{3}\right)$,

$q_{+} \equiv-\theta^{\prime}(+1 / 2)=-1$

$q_{-} \equiv-\theta^{\prime}(-1 / 2)=-1$.

Equations (38)-(40) coincide with Eqs. (16)-(18) of Bühler [1], as well as with Eqs. (21)-(23) of Weidman [2].

\section{References}

1. Bühler K (2003) Special solutions of Boussinesq-equations for free convection flows in a vertical gap. Heat Mass Transf 39:631-638

2. Weidman PD (2006) Convection regime flow in a vertical slot: continuum solutions from capped to open ends. Heat Mass Transf. Online first, February 2006 